\title{
Erratum: Dependence of Rydberg-state creation by strong-field ionization on laser intensity [Phys. Rev. A 98, 033415 (2018)]
}

\author{
L. Ortmann, C. Hofmann, and A. S. Landsman
}

(Received 18 December 2018; published 16 January 2019)

DOI: 10.1103/PhysRevA.99.019901

In the original paper, the initial time dependence as used in Ref. [1] was not correctly implemented. In the following, we want to correct this. For the sake of consistency, we now also use the theory presented in Ref. [2]. This reference was already used for the initial transverse velocity in our paper. In order to sample the initial times (ionization time spread), the adapted version as described in Ref. [3] was implemented. The intensity dependence of the time width is shown in Fig. 1 along with a power-law fit $a F_{0}^{c}$, which yields the new exponent $c=0.43$ (or 0.86 for the intensity). The new overall exponent of the nonadiabatic intensity dependence obtained in the area estimation approach [Eq. (9) in the original paper] reads

$$
N^{*} / N \propto \frac{1 / \sqrt{I}}{\sigma_{\perp} \sigma_{\phi}} \propto 1 / I^{0.5+0.84 / 4+0.43 / 2}=1 / I^{0.925}
$$

As one can see, the exponent has barely changed. Similarly, rerunning the classical trajectory Monte Carlo simulations with the corrected time spread according to [2] changes the result only slightly: As Fig. 2 shows, the new exponent extracted from the updated simulations is -0.95 .

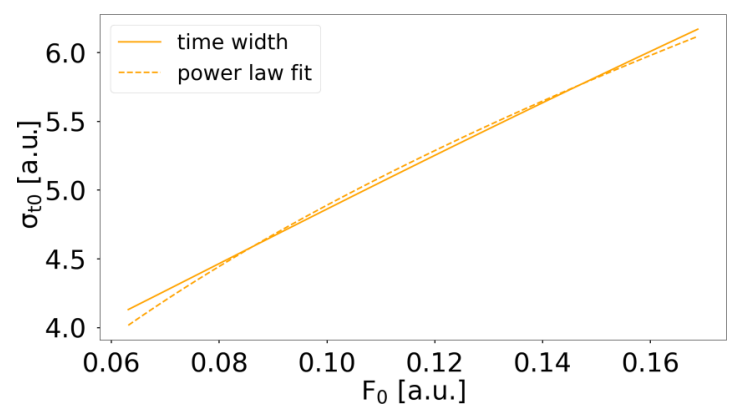

FIG. 1. Updated version of estimating the width of the ionization time distribution, thus, replacing the information formerly extracted from the curve $f$ in Fig. 2 in the original paper. The exponent extracted from the power-law fit is 0.43 . The widths $\sigma_{t_{0}}$ are obtained from fitting a Gaussian function with standard deviation $\sigma_{t_{0}}$ to the ionization time distribution given in Ref. [2].

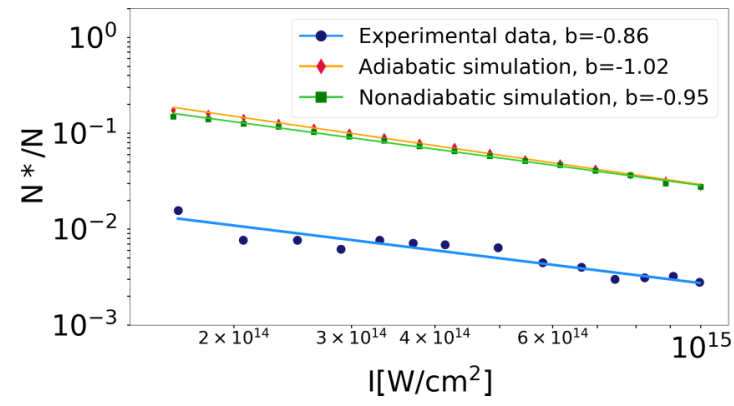

FIG. 2. Updated version of Fig. 1 in the original paper. 
In addition, the nonadiabatic power-law exponents for $\lambda=600$ and $\lambda=400 \mathrm{~nm}$ have to be updated. The new exponent for $\lambda=600 \mathrm{~nm}$ is $b=-0.83$ and for $\lambda=400 \mathrm{~nm}$ is $b=-0.48$.

Note that there are no changes to any of the conclusions made in the paper since only the exponent displayed in Fig. 2 changed slightly.

[1] D. I. Bondar, Phys. Rev. A 78, 015405 (2008).

[2] V. Mur, S. Popruzhenko, and V. Popov, J. Exp. Theor. Phys. 92, 777 (2001).

[3] C. Hofmann, A. S. Landsman, A. Zielinski, C. Cirelli, T. Zimmermann, A. Scrinzi, and U. Keller, Phys. Rev. A 90, 043406 (2014). 